University of New Orleans

ScholarWorks@UNO

\title{
A Trellis-Coded Modulation Scheme with a Novel Expanded 16-Dimensional Constant Envelope Q2PSK Constellation
}

\author{
Milton I. Quinteros \\ University of New Orleans \\ Edit J. Kaminsky \\ University of New Orleans, ejbourge@uno.edu \\ Kenneth V. Cartwright \\ College of The Bahamas
}

Follow this and additional works at: https://scholarworks.uno.edu/ee_facpubs

Part of the Electrical and Electronics Commons

\begin{abstract}
Recommended Citation
Quinteros, M. and E. Kaminsky, "A Trellis-Coded Modulation Scheme with a Novel Expanded 16-Dimensional Constant Envelope Q2PSK Constellation”, IEEE Globecom 2009, Hawaii, 30 Nov. - 4 Dec. 2009, 6 pp.
\end{abstract}

This Conference Proceeding is brought to you for free and open access by the Department of Electrical Engineering at ScholarWorks@UNO. It has been accepted for inclusion in Electrical Engineering Faculty Publications by an authorized administrator of ScholarWorks@UNO. For more information, please contact scholarworks@uno.edu. 


\section{A Trellis-Coded Modulation Scheme with a Novel Expanded 16-Dimensional Constant Envelope $\mathrm{Q}^{2} \mathrm{PSK}$ Constellation}

\author{
Milton I. Quinteros \\ Department of Electrical Engineering \\ EN 616A Lakefront Campus \\ University of New Orleans \\ New Orleans, LA 70148, U.S.A. \\ mquinter@uno.edu
}

\author{
Edit J. Kaminsky \\ Department of Electrical Engineering \\ EN 809A Lakefront Campus \\ University of New Orleans \\ New Orleans, LA 70148, U.S.A. \\ ejbourge@uno.edu
}

\author{
Kenneth V. Cartwright \\ School of Sciences and Technology \\ College of The Bahamas \\ P.O. Box N4912 \\ Nassau, N.P., Bahamas \\ kvc@batelnet.bs
}

\begin{abstract}
This paper presents a TCM scheme that uses a new expanded 16-Dimensional Constant Envelope $Q^{2} P S K$ constellation along with a simple convolutional encoder of rate 2/3. An effective gain of $2.67 \mathrm{~dB}$ over uncoded $C E Q^{2} P S K$ is achievable with low complexity, and without suffering from constellation expansion penalty. Larger coding gains are easily achieved with encoders of higher rates. In addition, an optimal hardware implementation of the required decoders is described.
\end{abstract}

Keywords-Multidimensional constellation, constant envelope, constellation expansion, trellis coded modulation, quadraturequadrature phase shift keying.

\section{INTRODUCTION}

Trellis-coded modulation schemes with multidimensional signals allow for performance improvement over classical twodimensional constellations. For example, in [1], [2] it was claimed that TCM systems with lattices of four-, eight-, or 16dimensions achieve decent coding gains of $2 \mathrm{~dB}, 3 \mathrm{~dB}$, or 6 $\mathrm{dB}$, respectively, over two-dimensional lattices but with a loss due to constellation expansion. Indeed, the disadvantage of the constellation expansion required to introduce coding redundancy in standard TCM is the reduction of the minimum squared Euclidian distance (MSED) between points for a given energy level, or the increase of modulation level and energy for a given MSED [3].

In [4], Saha and Arbor reported a set of signals that uses two data shaping pulses and two carriers which are pair-wise quadrature in phase to create a spectrally efficient four dimensional (4-D) signal set called Quadrature-Quadrature Phase Shift-Keying (Q $\left.{ }^{2} \mathrm{PSK}\right)$.

Acha and Carrasco [5] and Saha [6] utilize Saha's standard 4-D $\mathrm{Q}^{2} \mathrm{PSK}$ constellation for their TCM systems along with convolutional encoders of different rates. These schemes, however, achieve some gains at the cost of data rate. In addition to the rate cost paid for using these schemes, and the care required in order to avoid catastrophic error propagation [5], some of the $\mathrm{Q}^{2} \mathrm{PSK}$ trellis codes proposed by Saha, Acha and Carrasco do not have constant envelope. Their constant envelope TCM systems are obtained by further reducing the data rate by half.
During recent years, some work has been done in design of multidimensional signal sets that allow TCM to be implemented without constellation expansion penalty [3], [7], i.e., without increasing the modulation level. Kaminsky, Ayo and Cartwright's multidimensional TCM schemes of [3] are based on QPSK signals of even dimensions of eight and above. This family of constant envelope constellations is generated by concatenating $n$ QPSK points or $n$ QPSK points rotated by 45 degrees $(n \geq 4)$ without any constellation expansion loss. In [7], a 16-D signal set with constant envelope was generated by concatenating four $\mathrm{CEQ}^{2} \mathrm{PSK}$ signals from Saha's or four $\mathrm{CEQ}^{2} \mathrm{PSK}$ signals from Cartwright's 4-D constellation. Therefore, the same idea of [3] is followed in [7] to introduce redundancy for coding without increasing the modulation level while preserving average and peak energies constant.

Here, we use the constellation we proposed in [7] to implement a simple multidimensional TCM system that uses a convolutional encoder of rate $2 / 3$ to achieve an asymptotic coding gain of $3 \mathrm{~dB}$ over uncoded $\mathrm{CEQ}^{2} \mathrm{PSK}$. Because nonlinear channels require constant envelope signals, this 16-D $\mathrm{CEQ}^{2} \mathrm{PSK}-\mathrm{TCM}$ system is a good option in channels that require non-linear power amplifiers. Larger coding gains are easily achieved with this constellation by using higher-rate encoders.

Additionally, a hardware detector (based on the demodulator described in [8]) for the 4-D CEQ ${ }^{2} \mathrm{PSK}$ discovered by Cartwright is proposed here. The complete implementation of the TCM system is also given.

The rest of this paper is organized as follows: In Section II, a review of $\mathrm{CEQ}^{2} \mathrm{PSK}$ constellations and their decoders including presentation of our new hardware detector for Cartwright's CEQ ${ }^{2} \mathrm{PSK}$ - is presented. Section III presents the set-partitioning into eight sets required for the novel 16-D expanded $\mathrm{CEQ}^{2} \mathrm{PSK}$ constellation. Section IV discusses the TCM system implementation, and Section $V$ reports the development of the TCM decoder. Results, including Monte Carlo simulations of the system proposed in this paper are presented and discussed in Section VI. Finally, in Section VII, conclusions are drawn and future work is mentioned, followed by references. 


\section{REVIEW OF THE CONSTANT ENVELOPE Q ${ }^{2}$ PSK CONSTELLATIONS}

In this section we discuss separately the two 4-dimensional constant envelope $\mathrm{Q}^{2} \mathrm{PSK}$ constellations, and the expanded 16dimensional Q ${ }^{2} \mathrm{PSK}$ constellation.

A. 4-D Constant Envelope Quadrature-Quadrature Phase Shift-Keying (CEQ $\left.{ }^{2} P S K\right)$

In what follows, we review Saha's original 4-D CEQ ${ }^{2}$ PSK [9] and the 4-D CEQ ${ }^{2} \mathrm{PSK}$, discovered by Cartwright [7]. An optimal decoder for the former was presented in [8] and a similar hardware decoder for the latter is proposed here.

\section{1) Saha's 4-D CEQ $Q^{2} P S K$}

Quadrature-Quadrature Phase Shift-keying ( $\left.{ }^{2} \mathrm{PSK}\right)$ and Constant Envelope $Q^{2} \mathrm{PSK}$ (CEQ $\left.{ }^{2} \mathrm{PSK}\right)$ signal sets were introduced by Saha and Birdsall in [9].

The four dimensional non-constant envelope $\mathrm{Q}^{2} \mathrm{PSK}$ may be defined as

$$
S_{q}(t)=\sum_{i=1}^{4} a_{i}(t) s_{i}(t)
$$

where the four signals $\left\{a_{i}(t)\right\}, i=1, \ldots, 4$, each of duration $2 \mathrm{~T}$, are the original binary data streams, and the modulating signal set $\left\{s_{i}(t)\right\}, i=1, \ldots, 4$, is defined as follows [9]:

$$
\begin{aligned}
& s_{1}(t)=\cos (\pi t / 2 \mathrm{~T}) \cos \left(2 \pi f_{c} t\right),|t| \leq \mathrm{T} \\
& s_{2}(t)=\sin (\pi t / 2 \mathrm{~T}) \cos \left(2 \pi f_{c} t\right),|t| \leq \mathrm{T} \\
& s_{3}(t)=\cos (\pi t / 2 \mathrm{~T}) \sin \left(2 \pi f_{c} t\right),|t| \leq \mathrm{T} \\
& s_{4}(t)=\sin (\pi t / 2 \mathrm{~T}) \sin \left(2 \pi f_{c} t\right),|t| \leq \mathrm{T} .
\end{aligned}
$$

The carrier frequency, $f_{c}$, should be $\mathrm{n} /(4 \mathrm{~T})$ where $\mathrm{n} \geq 2$, and $\mathrm{T}$ is the time duration of 2 bits.

In order to obtain constant envelope, Saha and Birdsall introduced an encoder of rate $3 / 4$ that accepts three information serial input streams $\left\{a_{I}(t), a_{2}(t), a_{3}(t)\right\}$, and generates a code word $\left\{a_{1}(t), a_{2}(t), a_{3}(t), a_{4}(t)\right\}$ such that the first three bits in the codeword are the information bits and the fourth is an odd parity check bit [9]. Therefore, the eight possible transmitted signals for the original $\mathrm{CEQ}^{2} \mathrm{PSK}$ are $S_{1}=[a, a, b,-b]$ and $S_{2}=$ $[a,-a, b, b]$, where $a, b$ are either +1 or -1 [8]. It is also mentioned in [9] that $\mathrm{CEQ}^{2} \mathrm{PSK}$ is achieved at the expense of the information transmission rate which is reduced from $2 / \mathrm{T}$ to $3 /(2 \mathrm{~T})$.

To obtain the maximum achievable performance of $\mathrm{CEQ}^{2} \mathrm{PSK}$ an optimal detector is needed. In [8], Cartwright and Kaminsky presented a $\mathrm{CEQ}^{2} \mathrm{PSK}$ hardware detector that reaches the performance of $\mathrm{CEQ}^{2} \mathrm{PSK}$ predicted in [9]. This decoder uses five hard-limiters, four adders, four absolute value circuits, two inverters, and a decision function that activates a trigger for a four-pole double-throw switch.

\section{2) Cartwright's 4-D CEQ $Q^{2} P S K$}

In [7], a new set of eight 4-D symbols that is also valid for $\mathrm{CEQ}^{2} \mathrm{PSK}$ was introduced. This new set has the same energy and distribution of squared distances as the original $\mathrm{CEQ}^{2} \mathrm{PSK}$ constellation from [9]. Cartwright's symbols may be defined by an orthogonal transformation of Saha's constant envelope symbols. Let $\mathrm{R}^{4}$ be the 4-D rotational operation [10]:

$$
R^{4}=\left[\begin{array}{ll}
R & 0 \\
0 & R
\end{array}\right]
$$

where $R$ is

$$
R=\left[\begin{array}{cc}
\cos \left(45^{\circ}\right) & -\sin \left(45^{\circ}\right) \\
\sin \left(45^{\circ}\right) & \cos \left(45^{\circ}\right)
\end{array}\right] \text {. }
$$

Because the eight possible transmitted 4-D signals for Cartwright's constellations are generated by rotating the component 2-D signals, the new CEQ ${ }^{2} \mathrm{PSK}$ points, $S_{1 r}$ and $S_{2 r}$, corresponding to Saha's $S_{1}$ and $S_{2}$ are:

$$
\begin{aligned}
& S_{1 r}=R^{4} S_{1}, \\
& S_{2 r}=R^{4} S_{2},
\end{aligned}
$$

or $S_{1 r}=[0, \sqrt{2} a, \sqrt{2} b, 0]$ and $S_{2 r}=[\sqrt{2} a, 0,0, \sqrt{2} b]$, where $a, b$ are either +1 or -1 . The proof that these eight symbols are also valid for $\mathrm{CEQ}^{2} \mathrm{PSK}$ is given in [7].

We now discuss the implementation of the optimal hardware detector for Cartwright's constellation. Fig. 1 depicts the block diagram of our proposed detector which closely resembles the receiver in [8], but uses a different decision function $F(\cdot)$, gains of magnitude $\sqrt{2}$, and requires four multipliers which may be implemented as electronic switches, if so desired.

The received signal $r(t)$ is the transmitted signal $s(t)$ corrupted by additive white Gaussian noise (AWGN) $n(t)$ with power spectral density $\mathrm{N}_{\mathrm{o}}$ :

$$
r(t)=s(t)+n(t)
$$

The block F( $\cdot)$ in Fig. 1 calculates $\hat{d}$ as in (8):

$$
\hat{d}=\frac{1}{2}[\operatorname{sgn}(w-y)+1],
$$

and therefore determines the estimated symbol $\hat{S}=\left[\hat{a}_{1}, \hat{a}_{2}, \hat{a}_{3}\right.$, $\hat{a}_{4}$ ]. The values of $w$ and $y$ are given by (9) and (10), respectively:

$$
\begin{aligned}
& w=\left|a_{1 r}\right|+\left|a_{4 r}\right|, \\
& y=\left|a_{2 r}\right|+\left|a_{3 r}\right|,
\end{aligned}
$$

and $\left\{\mathrm{a}_{i r}\right\}, i=1, \ldots, 4$ are the outputs of the correlation detectors.

If a member of $S_{1 \mathrm{r}}$ is transmitted, $y=2 \sqrt{2}$ and $w=0$, but when a member of $S_{2 \mathrm{r}}$ is transmitted, $w=2 \sqrt{2}$ and $y=0$. Therefore, when a member of $S_{1 \mathrm{r}}$ has been transmitted $w<y$ and $\hat{d}=0$, but when $w>y$, a member of the $S_{2 \mathrm{r}}$ has been transmitted and $\hat{d}=1$. The output symbol, then, is obtained from (9):

$$
\hat{S}=\hat{d} \hat{S}_{2 r}+(1-\hat{d}) \hat{S}_{1 r} .
$$

Our optimum hardware decoder is a direct implementation of

$$
\begin{aligned}
& \hat{a}_{l r}=\sqrt{2} \operatorname{sgn}\left(a_{1 r}\right) \hat{d} \\
& \hat{a}_{2 r}=\sqrt{2} \operatorname{sgn}\left(a_{2 r}\right)(1-\hat{d}) \\
& \hat{a}_{3 r}=\sqrt{2} \operatorname{sgn}\left(a_{3 r}\right)(1-\hat{d}) \\
& \hat{a}_{4 r}=\sqrt{2} \operatorname{sgn}\left(a_{4 r}\right) \hat{d},
\end{aligned}
$$

which follows from (11).

In order to verify the performance of the demodulator, Monte Carlo simulations were performed and compared with 


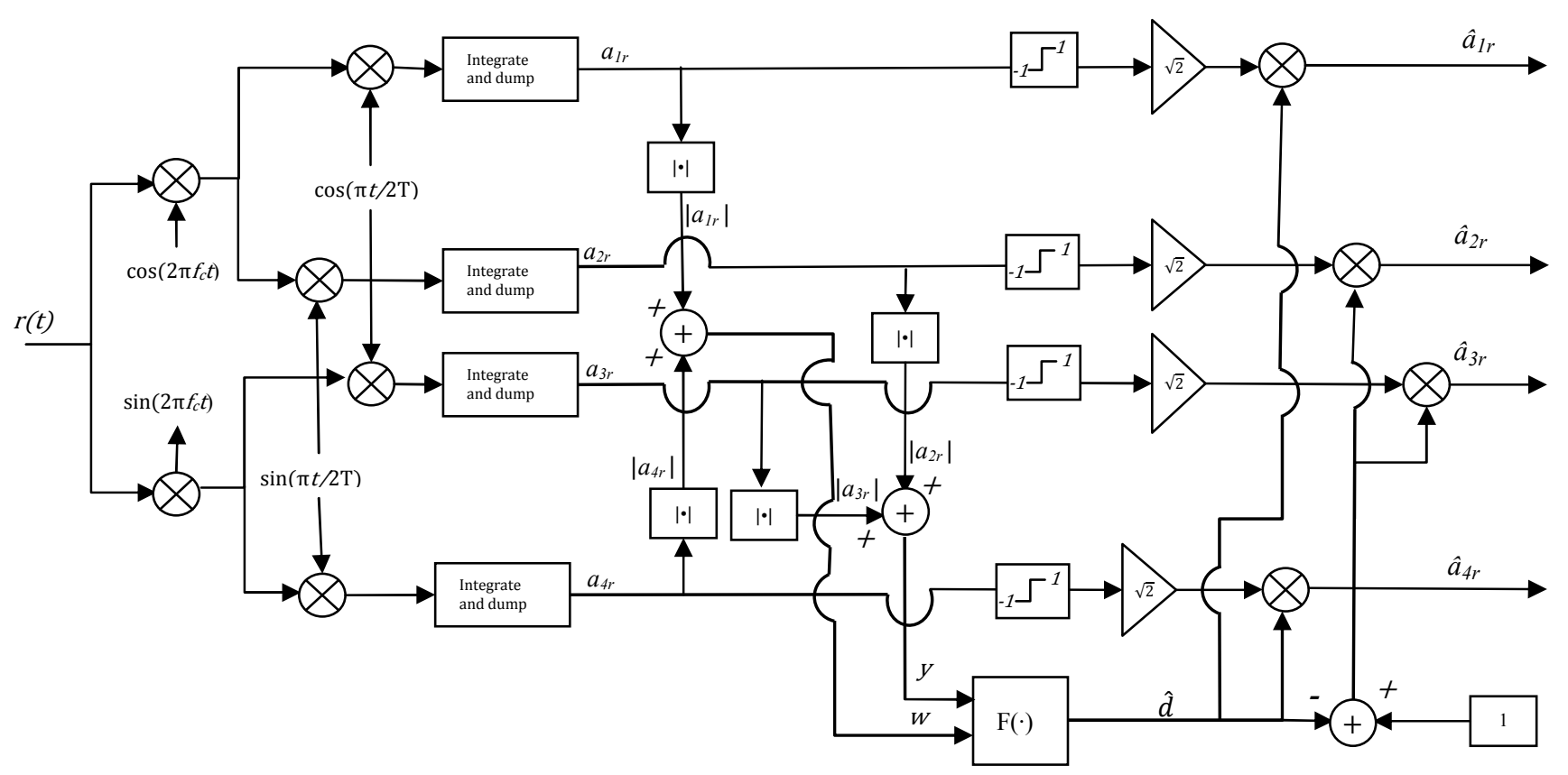

Fig. 1: Block diagram of the proposed optimum 4-D CEQ $Q^{2} P S K$ demodulator for Cartwright's signal constellation.

the optimum hardware detector published in [8]; these results are presented in Section VI.

\section{B. Novel 16-D Expanded CEQ $Q^{2} P S K$ Constellation}

If four consecutive 4-D points from either Saha's or Cartwright's $\mathrm{CEQ}^{2} \mathrm{PSK}$ constellation are taken together, a 16D signal is obtained. In this way, two sets of 4096 16-D symbols each, $S_{a}$ for Saha's or $S_{b}$ for Cartwright's, are formed. The expanded constellation, $V$, is defined as the union of these two constellations:

$$
V=\left\{S_{a} \cup S_{b}\right\}
$$

Our expanded 16-D CEQ ${ }^{2}$ PSK signal set is therefore formed in a way similar to Kaminsky, Ayo and Cartwright's expanded constellation of [3], but with different constituent signal points. Peak energy, average energy, and minimum squared Euclidian distance (MSED) is maintained while doubling the size of the constellation, so this set of 16-D symbols is obtained without any constellation expansion penalty. Because the four consecutive 4-D symbols must come from one or the other 4-D $\mathrm{CEQ}^{2} \mathrm{PSK}$ constellation, the set-partition for the TCM system cannot be performed exactly as is done when the expanded constellation is formed by the Cartesian product of the constituent constellations, as in [1]. In the next Section we present the set partition for the 16-D expanded constellation $V$.

\section{EXPANDED 16-D CEQ ${ }^{2}$ PSK CONSTELLATION PARTITION}

TCM schemes require a proper set-partitioning of the constellation in order to increment the free distance of the code. In this section we show how the constellation $V$ is partitioned into the eight subsets required by our simple TCM encoder. We use $\left\{A_{i}\right\}_{i=1, \ldots, 4}$ to denote the four subsets formed from $S_{a}$ and $\left\{\mathrm{B}_{\mathrm{i}}\right\}_{\mathrm{i}=1, \ldots, 4}$ for the 16-D CEQ ${ }^{2} \mathrm{PSK}$ points from $S_{b}$. The MSED within $V$ is 8 , but the intra-subset MSED within
$\left\{A_{i}\right\}$ or $\left\{B_{i}\right\}$ is increased to 16 . This allows us to achieve an asymptotic gain of $3 \mathrm{~dB}$ with just 8 subsets and a simple 8state convolutional encoder of rate $2 / 3$. To achieve larger gains, further partitioning is needed, along with a trellis with more states.

First, each family $S_{a}$ and $S_{b}$ is partitioned independently by using the method of Wei [1], as follows: The 4-D constituent points of the set $S_{a}$ (the eight original CEQ ${ }^{2} \mathrm{PSK}$ signals of Saha [9]) $\left\{S_{1} \cup S_{2}\right\}$ can be partitioned into eight sublattices named $1,2,4,8,14,13,11,7$ (to correspond to their binary values). The same is true for $\left\{\mathrm{S}_{1 \mathrm{r}} \cup \mathrm{S}_{2 \mathrm{r}}\right\}$, the constituent 4-D points of the set $S_{b}$, but the eight sublattices are named $1_{\mathrm{r}}, 2_{\mathrm{r}}, 4_{\mathrm{r}}, 8_{\mathrm{r}}, 14_{\mathrm{r}}, 13_{\mathrm{r}}, 11_{\mathrm{r}}, 7_{\mathrm{r}}$. Now we have 164 -D sublattices with MSED of 8; these are shown in Table I.

Next, we group these 164 -D sublattices into 8 groups of antipodal signals. These groups are called $Q_{i}$ for Saha's and $Q_{i r}$ for Cartwright's signals, and $i=1, \ldots, 4$. Table II shows these groups. At this point, we have reduced the number of sublattices from 16 to 8 , and we have increased the MSED within $Q_{i}$ and $Q_{i r}$ to 16 . Each $\mathrm{Q}$ group has two 4-D signals.

We now form the 8-D types by concatenating two 4-D $Q_{i}$ or two 4-D $Q_{i r}$ to obtain 32 8-D types with MSED of 8 . These 32 types are defined as $Q_{i j}=\left[Q_{i}, Q_{j}\right]$ and $Q_{i j r}=\left[Q_{i r}, Q_{j r}\right], i, j=1, \ldots$, 4. We now proceed to group the $Q_{i j}$ and $Q_{i j r}$ into eight 8-D sets $W_{i}$ and $W_{i r}$ of 16 points each, such that the intra-set MSED is equal to 16 . This grouping is shown in Table III.

To proceed further, from the $W$ types we construct the 16-D sublattices by concatenating two 8-D types: $W_{i j}=\left[W_{i}, W_{j}\right]$, and $W_{i j r}=\left[W_{i r}, W_{j r}\right], i, j=1, \ldots, 4$. These 32 16-D sublattices have MSED of 16 and 256 points each.

Finally, these 16-D $W_{i j}$ and $W_{i j r}$ sublattices are grouped into the eight subsets $\left\{\mathrm{A}_{k}\right\},\left\{\mathrm{B}_{k}\right\}, k=1, \ldots, 4$. Table IV shows how the $W_{i j}$ and $W_{i j r}$ are grouped. These subsets still have MSED of 


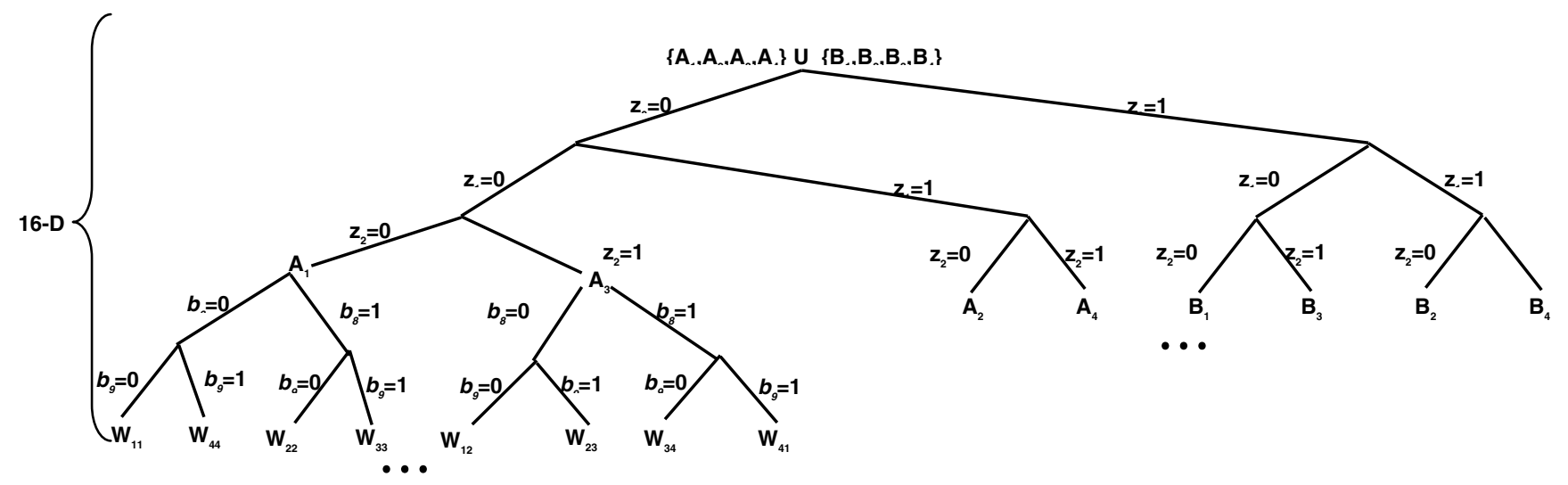

Fig. 2: Partition of the 16-D Constant Envelope $Q^{2} P S K$ constellation $V$.

16, contain 1024 points each, and they are required for the TCM system that uses a convolutional encoder of rate $2 / 3$. Fig. 2 shows a tree diagram of the set partitioning.

\section{TCM SYSTEM IMPLEMENTATION}

Our multidimensional TCM system uses one of Ungerboeck's feedback convolutional encoders from [11]. It has rate $2 / 3$ and constraint length 3 and is shown in Fig. 3. Remember that our TCM system has a $\mathrm{CEQ}^{2} \mathrm{PSK}$ modulator over four consecutive modulation time intervals, each of duration $2 \mathrm{~T}$.

Fig. 4 depicts the complete 16-D CEQ ${ }^{2}$ PSK-TCM system. Two of the 12 bits of information, $\left(b_{10}, b_{11}\right)$, arriving every four signaling intervals enter the convolutional encoder to produce

Table I: The 4-D CEQ ${ }^{2}$ PSK points

\begin{tabular}{|c|c|c|c|c|c|c|}
\hline $\mathrm{S}_{1} \cup \mathrm{S}_{2}$ & Saha's & $\mathrm{S}_{1 \mathrm{r}} \cup \mathrm{S}_{2 \mathrm{r}}$ & \multicolumn{4}{|c|}{ Cartwright's } \\
\hline 1 & $\begin{array}{llll} & -1 & -1 & 1\end{array}$ & $1_{\mathrm{r}}$ & 0 & $-\sqrt{2}$ & $-\sqrt{2}$ & 0 \\
\hline 2 & $\begin{array}{llll}-1 & -1 & 1 & -1\end{array}$ & $2 \mathrm{r}$ & 0 & $-\sqrt{2}$ & $\sqrt{2}$ & 0 \\
\hline 4 & $\begin{array}{llll}-1 & 1 & -1 & -1\end{array}$ & $4_{\mathrm{r}}$ & $-\sqrt{2}$ & 0 & 0 & $-\sqrt{2}$ \\
\hline 8 & $1-1-1-1$ & $8 \mathrm{r}$ & $\sqrt{2}$ & 0 & 0 & $-\sqrt{2}$ \\
\hline 14 & $\begin{array}{llll}1 & 1 & 1 & -1\end{array}$ & $14 \mathrm{r}$ & 0 & $\sqrt{2}$ & $\sqrt{2}$ & 0 \\
\hline 13 & $\begin{array}{llll}1 & 1 & -1 & 1\end{array}$ & $13 \mathrm{r}$ & 0 & $\sqrt{2}$ & $-\sqrt{2}$ & 0 \\
\hline 11 & $\begin{array}{llll}1 & -1 & 1 & 1\end{array}$ & $11_{r}$ & $\sqrt{2}$ & 0 & 0 & $\sqrt{2}$ \\
\hline 7 & $\begin{array}{llll}-1 & 1 & 1 & 1 \\
\end{array}$ & $7 \mathrm{r}$ & $-\sqrt{2}$ & 0 & 0 & $\sqrt{2}$ \\
\hline
\end{tabular}

Table II: Grouping of the 4-D constituent points into sets of antipodal signals

\begin{tabular}{|cl|}
\hline Saha's Q & Cartwright's $\mathrm{Q}_{\mathrm{r}}$ \\
\hline$Q_{1}=\{1 ; 14\}$ & $Q_{1 r}=\left\{1_{r} ; 14_{r}\right\}$ \\
$Q_{2}=\{2 ; 13\}$ & $Q_{2 r}=\left\{2_{r} ; 13_{r}\right\}$ \\
$Q_{3}=\{4 ; 11\}$ & $Q_{3 r}=\left\{4_{r} ; 11_{r}\right\}$ \\
$Q_{4}=\{8 ; 7\}$ & $Q_{4 r}=\left\{8_{r} ; 7_{r}\right\}$ \\
\hline
\end{tabular}

Table III: 8-D groups $W$

\begin{tabular}{|cc|}
\hline Saha's W & Cartwright's W $\mathrm{W}_{\mathrm{r}}$ \\
\hline$W_{1}=\left\{Q_{11} ; Q_{22} ; Q_{33} ; Q_{44}\right\}$ & $W_{1 r}=\left\{Q_{11 r} ; Q_{22 r} ; Q_{33 r} ; Q_{44 r}\right\}$ \\
$W_{2}=\left\{Q_{12} ; Q_{23} ; Q_{34} ; Q_{41}\right\}$ & $W_{2 r}=\left\{Q_{12 r} ; Q_{23 r} ; Q_{34 r} ; Q_{41 r}\right\}$ \\
$W_{3}=\left\{Q_{13} ; Q_{24} ; Q_{31} ; Q_{42}\right\}$ & $W_{3 r}=\left\{Q_{13 r} ; Q_{24 r} ; Q_{31 r} ; Q_{42 r}\right\}$ \\
$W_{4}=\left\{Q_{14} ; Q_{21} ; Q_{32} ; Q_{43}\right\}$ & $W_{4 r}=\left\{Q_{14 r} ; Q_{21 r} ; Q_{32 r} ; Q_{43 r}\right\}$ \\
\hline
\end{tabular}

Table IV: Final grouping of the 16-D CEQ ${ }^{2} \mathrm{PSK}$ signals

\begin{tabular}{|cc|}
\hline Saha's A & Cartwright's B \\
\hline$A_{1}=\left\{W_{11} ; W_{22} ; W_{33} ; W_{44}\right\}$ & $B_{1}=\left\{W_{11 r} ; W_{22 r} ; W_{33 r} ; W_{44 r}\right\}$ \\
$A_{2}=\left\{W_{12} ; W_{23} ; W_{33} ; W_{41}\right\}$ & $B_{2}=\left\{W_{12 r} ; W_{23 r} ; W_{34 r} ; W_{41 r}\right\}$ \\
$A_{3}=\left\{W_{13} ; W_{24} ; W_{31} ; W_{42}\right\}$ & $B_{3}=\left\{W_{13 r} ; W_{24 r} ; W_{31 r} ; W_{42 r}\right\}$ \\
$A_{4}=\left\{W_{14} ; W_{21} ; W_{32} ; W_{43}\right\}$ & $B_{4}=\left\{W_{14 r} ; W_{21 r} ; W_{32 r} ; W_{43 r}\right\}$ \\
\hline
\end{tabular}

three coded bits $\left(z_{0}, z_{1}, z_{2}\right)$. The output of the convolutional encoder selects one of the eight subsets obtained in Section III, $\mathrm{A}_{k}$ or $\mathrm{B}_{k}$. Two other uncoded bits $\left(b_{8}, b_{9}\right)$ select one of the $W_{i j}$ or $W_{i j r}$ types from within the selected group. Fig. 2 shows the mapping of these five bits to some of the 16-D subsets.

Finally, the rest of the information bits $\left(b_{0}\right.$ through $\left.b_{7}\right)$ select one of the 256 points from within the selected 16-D $W_{i j}$ or $W_{i j r}$ types. The selected signal $s(t)$ is transmitted. In the next Section we discuss the required decoding for the modulation scheme aforementioned.

\section{TCM DECODING}

In our TCM system, the received signals, corrupted by noise, are decoded by using a soft-decision maximum-likelihood sequence decoder [12]. We use the Viterbi decoding algorithm [13], [14] to search the trellis and find the most likely paths, given the received sequence of subsets. The trellis is shown in Fig. 5 with the subset assignment given in the usual top-down fashion. Because our convolutional encoder has a constraint length of 3 and rate $2 / 3$, a decoding depth of 24 was used in the decoder implementation [14].

Fig. 6 shows a simplified block diagram of the decoder used in our $16-\mathrm{D} \mathrm{CEQ}^{2} \mathrm{PSK}-\mathrm{TCM}$ system. The noisy signal, $r(t)$, goes simultaneously to the decoders for Saha's and Cartwright's CEQ ${ }^{2} \mathrm{PSK}$, but without implementing the hardlimitting operations. These soft 16-D output symbols are the input to the VA decoder.

The mapping from input symbols to output bits is performed as follows: First, the VA estimates the most likely of the state transitions and the corresponding subset for that transition after 24 16-D intervals of modulation; therefore, by using the state transitions and the subset, the two information bits $\left(b_{10}, b_{11}\right)$ can be decoded. Finally, the other 10 bits are obtained by using a look-up table of 1024 rows, corresponding to the 1024 symbols in the estimated subset.

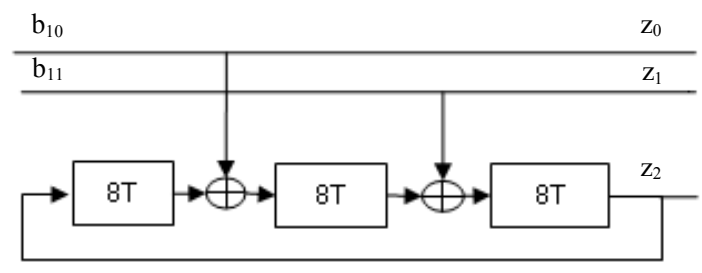

Fig. 3: Convolutional encoder of rate 2/3. 


\section{RESULTS}

We divide this Section into three parts. First, we discuss the results of our proposed hardware detector for Cartwright's 4-D $\mathrm{CEQ}^{2} \mathrm{PSK}$ constellation, presented in Section II. Then we present the analysis of the gains for our coded 16-D $\mathrm{CEQ}^{2} \mathrm{PSK}-\mathrm{TCM}$ system over the uncoded reference CEQ ${ }^{2} \mathrm{PSK}$ system $\left(S_{a}\right.$ or $\left.S_{b}\right)$, and the required information about the distance distribution of the appropriate constellations. Finally, we present and briefly discuss the simulation results which corroborate our analysis.

\section{A. Results for the Hardware Detector for Cartwright's 4-D $Q^{2} P S K$ Constellation}

Fig. 7 shows the performance of our hardware detector for Cartwright's 4-D $\mathrm{CEQ}^{2} \mathrm{PSK}$ constellation in terms of probability of bit error versus bit signal to noise ratio $\left(E_{b} / N_{o}\right)$, with $E_{b}$ the energy per information bit and $N_{o}$ the spectral density of the Additive White Gaussian Noise (AWGN). The markers indicate simulation results and the line shows the theoretical results [8]. Monte Carlo simulations for our hardware decoder were run until 50 errors were counted and match the results reported in [8] for the standard $\mathrm{CEQ}^{2} \mathrm{PSK}$ signal set detector and also match the theoretical results.

\section{B. Distance Properties, Coding Gains and Complexity}

Table $\mathrm{V}$ lists the smallest twelve squared Euclidian distances (SED) of the expanded $\mathrm{CEQ}^{2} \mathrm{PSK}$ constellation. Table VI shows the SED distribution of the partitioned constellation. The columns labeled $d_{k}^{2}$ represent the SED, and the values in the column named $\mathrm{N}\left(d_{k}\right)$ are the number of points at SED $d_{k}^{2}$. The MSED for the uncoded constellation (CEQ ${ }^{2} \mathrm{PSK}$ ) is $d_{u}^{2}=8$, and has $N_{u}=24$ points at that distance. The free distance of our simple TCM system is given by the parallel transitions in the trellis and is $d_{c}^{2}=16$ with an error coefficient (in 16-D) of $N_{c}=76$. These values determine the asymptotic gain of the coded system [11], [12]:

$$
G_{a}=10 \log _{10}\left(\frac{d_{c}^{2}}{d_{u}^{2}}\right),
$$

which yields to $3.01 \mathrm{~dB}$ because the squared free distance is doubled. However, we also have to take into consideration the loss caused by the number of neighbors at MSED [3], [10]; this loss normalized to $2-\mathrm{D}, \lambda$, is [8]:

$$
\lambda=\frac{\log _{10}\left(\frac{N c}{N u}\right)}{\log _{10}(32)},
$$

which gives a loss of $0.33 \mathrm{~dB}$ for our code. The effective gain is therefore $\gamma_{e f f}=G_{a}-\lambda=2.67 \mathrm{~dB}$. Higher gains are possible with encoders of higher rate; the achievable asymptotic gains are also listed in Table $\mathrm{V}$.

Table V: SED of the expanded 16-D CEQ ${ }^{2} \mathrm{PSK}$

\begin{tabular}{|cc|cc|}
\hline$d_{k}^{2}$ & \multicolumn{1}{c}{$G_{a}(d B)$} & $d_{k}^{2}$ & $G_{a}(d B)$ \\
\hline 8.000 & - & 26.343 & 5.17 \\
9.373 & - & 32.000 & 6.02 \\
15.029 & - & 37.657 & 6.73 \\
16.000 & 3.01 & 40.000 & 6.99 \\
20.686 & 4.13 & 43.314 & 7.34 \\
24.000 & 4.77 & 48.000 & 7.78 \\
\hline
\end{tabular}

Table VI: SED distribution after set-partitioning

\begin{tabular}{|cc|}
\hline \multicolumn{2}{|c|}{ Subsets $\mathrm{A}_{\mathrm{i}}$ or $\mathrm{B}_{\mathrm{i}}$} \\
$d_{k}^{2}$ & $N\left(d_{k}\right)$ \\
\hline 16 & 76 \\
24 & 192 \\
32 & 486 \\
40 & 192 \\
48 & 76 \\
64 & 1 \\
\hline
\end{tabular}

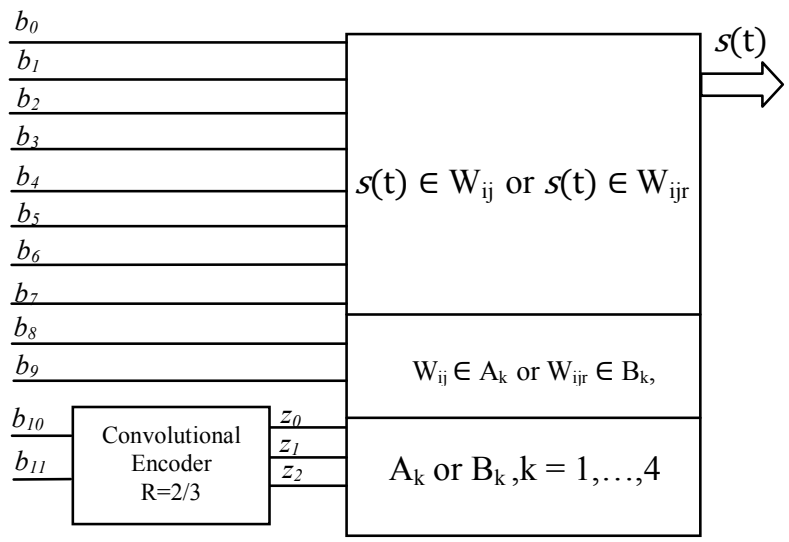

Fig. 4: Block diagram of the encoder/modulator for the proposed 16-D CEQ $Q^{2} P S K-T C M$ system.

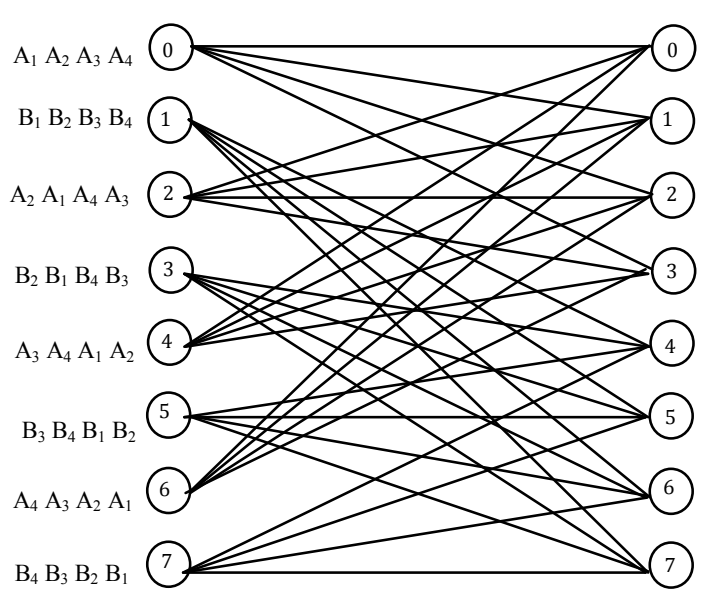

Fig. 5: Eight-state trellis and subset to branch assignments used for our CE $Q^{2} P S K-T C M$ system.

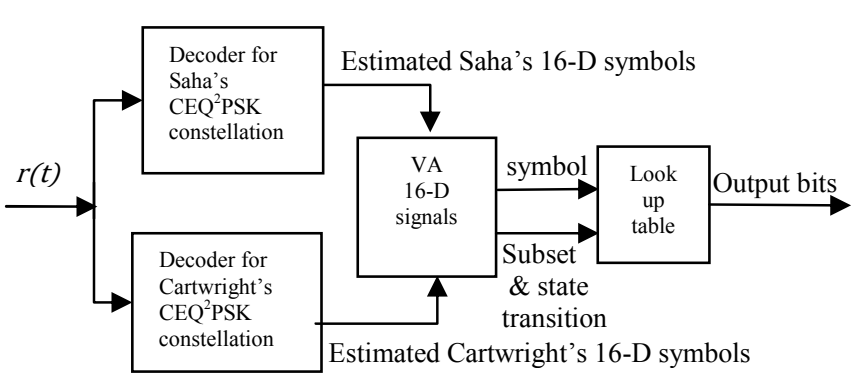

Fig. 6: Functional block of the decoder for CEQ $Q^{2} P S K-T C M$ system. 


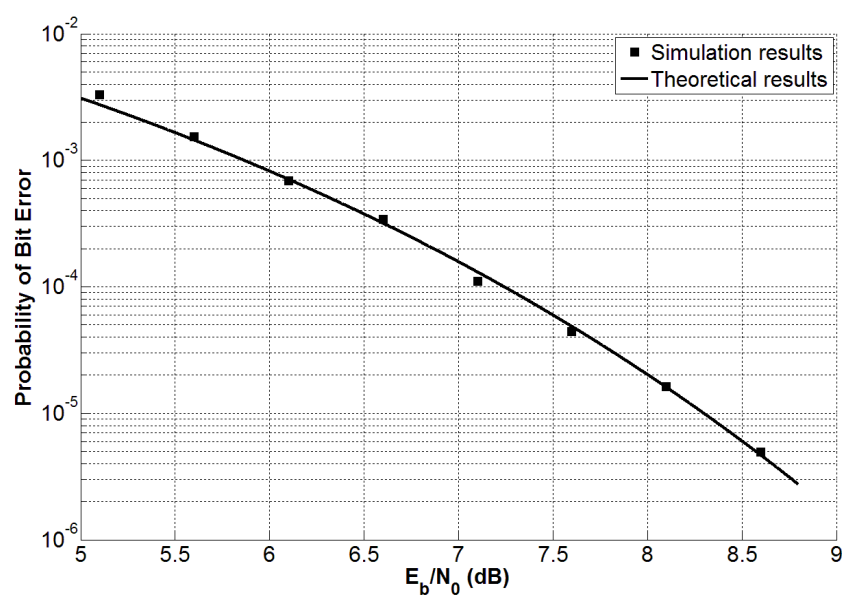

Fig. 7: Probability of bit and symbol error vs. $\mathrm{E}_{\mathrm{b}} / \mathbf{N}_{0}$ for our hardware detector for Cartwright's 4-D CEQ $Q^{2}$ PSK Constellation.

The decoding complexity of the TCM system presented here, as defined by Wei in [1], is $\beta=8$.

\section{TCM System Simulation Results}

The performance of our multidimensional TCM system was corroborated by using Monte Carlo Simulations; 20 errors are counted before the simulation stops. Fig. 8 shows the results in terms of bit and symbol error probabilities versus signal to noise ratio (SNR) for the reference uncoded $16-\mathrm{D} \mathrm{CEQ}^{2} \mathrm{PSK}$ and the trellis-coded 16-D system that uses the expanded $\mathrm{CEQ}^{2} \mathrm{PSK}$ constellation and decoding depth of 24. Comparison of the curves corresponding to the coded and uncoded probabilities of symbol error indicates that the effective gain of 2.67 is not yet achieved at a SNR of slightly over $8 \mathrm{~dB}$; the gain, however, increases with increasing SNR, and also with increasing decoding depth. The gain in bit error rate (BER) is slightly less because it cannot be guaranteed that a single bit is in error if a symbol is in error; as SNR increases, the likelihood of a single bit error per symbol error increases, so the bit and symbol probability of error curves tend to merge at large SNR.

\section{CONCLUSIONS AND FURTHER WORK}

The main contribution of this paper was to show the design of a TCM system using an expanded 16-D CEQ ${ }^{2}$ PSK constellation that allows the introduction of 1 bit of redundancy without constellation expansion penalty. We used a simple convolutional encoder of rate $2 / 3$ to achieve an effective gain of $2.67 \mathrm{~dB}$ while maintaining constant envelope and without reducing the bandwidth efficiency over the uncoded $\mathrm{CEQ}^{2} \mathrm{PSK}$ reference system. Considerably higher gains may be obtained with the same constellation by using more complex encoders. We also presented a hardware detector for Cartwright's 4-D Q ${ }^{2} \mathrm{PSK}$ constellation which was shown to be optimum.

Future work will include an analysis of the actual bandwidth efficiency of the system. The effects of non-linearities in the channel will be incorporated into the study, and the performance in fading channels will also be evaluated.

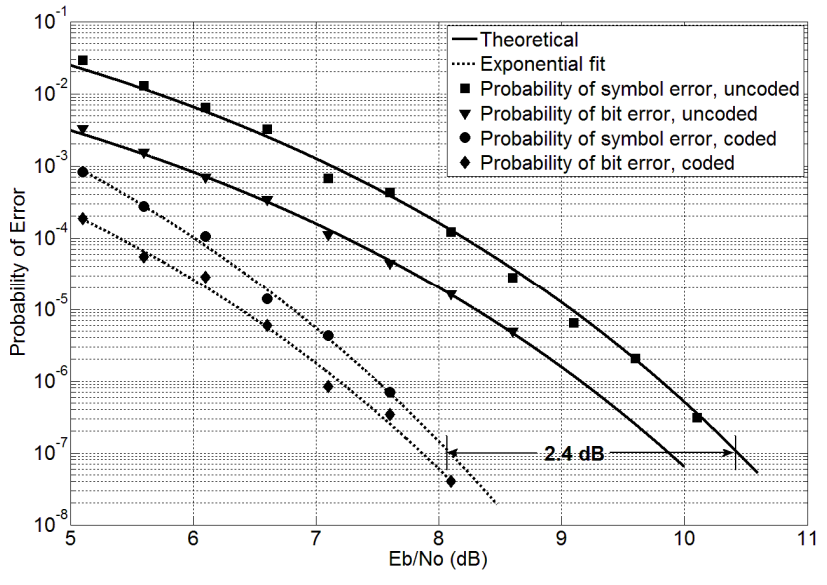

Fig.8: Bit and symbol error probabilities as a function of $\mathrm{E}_{\mathrm{b}} / \mathrm{N}_{\mathrm{o}}$ for coded and uncoded 16-D CEQ $Q^{2} P S K$ systems

\section{REFERENCES}

[1] L. F. Wei, "Trellis-Coded Modulation with Multidimensional Constellations," IEEE Trans. Inf. Theory, vol. IT-33, no. 4, pp. 483-501, Jul. 1987.

[2] G. D. Forney Jr., R. G. Gallager, G. R. Lang, F. M. Longstaff, and S. U. Qureshi, "Efficient Modulation for Band-Limited Channels," IEEE J. Select. Areas in Commun., vol. JSAC-2, no. 5, pp. 632-647, Sep. 1984.

[3] E. J. Kaminsky, J. Ayo, and K. V. Cartwright, "TCM without Constellation Expansion Penalty," J. Communications and Networks, vol. 4, no. 2, pp. 90-96, Jun. 2002.

[4] D. Saha and A. Arbor, "Quadrature-quadrature Phase Shift Keying," U.S. Patent 4730344, Mar. 8, 1988.

[5] V. Acha and R. A. Carrasco, "Trellis Coded Q ${ }^{2}$ PSK Signals. Part 1: AWGN and Nonlinear Satellite Channels," IEE Proc. Commun., vol. 141, no. 3, pp. 151-158, Jun. 1994.

[6] D. Saha, "Channel Coding with Quadrature-quadrature Phase ShiftKeying Signals," IEEE Trans. Commun., vol. 38, no. 4, pp. 409-501, Apr. 1990.

[7] M. I. Quinteros, K. V. Cartwright, E. J. Kaminsky, and R. U. Gallegos, "A Novel Expanded 16-Dimensional Constant Envelope Q ${ }^{2}$ PSK Constellation," in 2008 IEEE Region 5 BASICS2 Conf. Proc., Kansas City, MO, pp. 1-4, Apr. 2008.

[8] K. V. Cartwright and E. J. Kaminsky, "An Optimum Hardware Detector for Constant Envelope Quadrature-quadrature Phase Shift-Keying (CEQ ${ }^{2}$ PSK)," in IEEE Globecom 2005 Conf. Proc., vol. 1, St. Louis, MO, 2005, pp. 393-396, Dec. 2005.

[9] D. Saha and T. G. Birdsall, "Quadrature-Quadrature Phase Shift Keying," IEEE Trans. Commun., vol. 37, no. 4, pp. 437-448, May 1989.

[10] G. D. Forney, "Coset Codes Part 1: Introduction and Geometrical Classification," IEEE Trans. Inf. Theory, vol. 34, no. 5, pp. 1123-1151, Sep. 1988.

[11] G. Ungerboeck, "Channel Coding with Multilevel/Phase Signals ," IEEE Trans. Inf. Theory, vol. IT-28, no. 1, pp. 55-67, Jan. 1982.

[12] G. Ungerboeck, "Trellis-Coded Modulation with Redundant Signal Sets Part I and II," IEEE Commun. Mag., vol. 25, no. 2, pp. 5-21, Feb. 1987.

[13] A. J. Viterbi, "Error Bounds for Convolutional Codes and an Asymptotically Optimum Decoding Algorithm," IEEE Trans. Inf. Theory, vol. IT-13, no. 2, pp. 260-269, Apr. 1967.

[14] G. C. Clark and J. B. Cain, "Convolutional Code Structure and Viterbi Decoding," in Error-Correction Coding for Digital Communications. New York, United States: PlenumPress, 1981, pp. 262-263. 